\title{
TRANSFORMAÇÃO MALIGNA DE ADENOMA HEPÁTICO: DIAGNÓSTICO INCIDENTAL APÓS O TRANSPLANTE DE FÍGADO.
}

\author{
Malignant transformation of hepatocellular adenoma: post-liver transplantation incidental diagnosis.
}

\author{
Renato Ferreira da Silva, William José Duca, Paulo César Arroyo Jr, Raphael Raphe, Dalísio De Santi-Neto, Érica Fugikaha, Helen \\ Catarina Camarero Felício, Márcia Fumiê da Rocha e Rita de Cássia Martins Alves da Silva.
}

\begin{abstract}
RESUMO
O adenoma hepatocelular é um tumor hepático benigno, raro e geralmente solitário. Sua maior prevalência ocorre em mulheres que fazem uso de anticoncepcionais orais. Pode manifestar-se com dor no hipocôndrio direito, porém, na maioria dos pacientes é assintomático. As complicações mais temíveis são: hemorragia livre em peritônio por ruptura tumoral e degeneração maligna para carcinomahepatocelular (CHC). Descrevemos um caso de paciente com história de uso prolongado de anticoncepcional, que apresentava adenomas múltiplos, tendo indicação de laparotomia para ressecção cirúrgica. Ultrassom intra-operatório mostrou presença de esteatose hepática grau III e inúmeros adenomas que contra-indicaram a ressecção. Após acompanhamento ambulatorial por mais de dois anos, foi indicado transplante de fígado em razão da contra-indicação de ressecção dos adenomas associada a desconforto abdominal e baixa qualidade de vida. Foram encontrados incidentalmente focos de CHC em três adenomas no fígado explantado. Não havia aumento de alfa feto proteína ou evidencias radiológicas que sugerissem CHC. Embora transplante de fígado seja conduta radical e de exceção, pode ser usado em casos selecionados para tratamento de adenomas múltiplos que não permita a cirurgia de ressecção.
\end{abstract}

Descritores: Adenoma de Células Hepáticas; Carcinoma Hepatocelular; Transplante de Fígado.

\section{Instituições:}

Unidade de Cirurgia e Transplante de fígado e intestino Hospital de Base da Faculdade de Medicina de São José do Rio Preto - FUNFARME/ FAMERP.

\section{Correspondência:}

Renato Ferreira da Silva

Unidade de Cirurgia e Transplante de fígado e intestino do Hospital de Base da Faculdade de Medicina de São José do Rio Preto - FUNFARME/ FAMERP.

Av. Brigadeiro Faria Lima, 5416, CEP: 15090-090, São José do Rio Preto - SP.

Fone: (17) 3227-7030

E-mail: renatofsbr@gmail.com

Recebido em: 29.03.2009

Aceito em: 30.09 .2009

\section{INTRODUÇÃO}

$\mathrm{O}$ adenoma hepatocelular é um tumor benigno raro, mais freqüentemente identificado em mulheres em idade fértil e em uso de anticoncepcionais orais, sendo o uso de estrógenos o maior fator de risco para desenvolvimento desse tumor. ${ }^{1,2,3}$ Eventualmente, pode ser observado em homens em uso de esteróides anabolizantes ou andrógenos. ${ }^{1,4}$ Outros grupos de risco para desenvolvimento de adenomas hepáticos são: pacientes com glicogenose do tipo I ou Doença de Von Gierke, glicogenose tipo IV e diabetes. ${ }^{5,6}$ A maioria dos pacientes é assintomática e tem provas de função hepática e alfa-fetoproteína normais. ${ }^{3}$ Não existe exame de imagem que faça diagnóstico de certeza desse tumor, como ocorre, por exemplo, com a Hiperplasia Nodular Focal. O diagnóstico e classificação do tipo de adenoma só podem ser realizados através de biópsia. ${ }^{7,}$ Por ser o adenoma hipervascularizado, a biópsia percutânea deve ser evitada, e, quando necessária, deve ser feita por laparoscopia ou laparotomia. Dependendo da localização do tumor, alguns autores recomendam ressecção cirúrgica e estudo histológico da peça. ${ }^{3,9}$ Os grandes adenomas podem manifestar-se por dor vaga, desconforto no hipocôndrio direito e algumas vezes pode apresentar-se por quadro de choque hipovolêmico determinado pela ruptura espontânea do tumor..$^{10}$ Apesar de algumas lesões regredirem com a retirada do estrógeno ou permanecerem estáveis, o risco de ruptura com hemorragia e de transformação maligna para hepatocarcinoma existe. ${ }^{1,3,6,11,12}$ A cirurgia deve ser indicada sempre que possível e o transplante de fígado $(\mathrm{TxF})$ é uma opção terapêutica de exceção quando for impossível a ressecção cirúrgica. ${ }^{13-16,9,17}$

Relatamos a seguir a evolução de uma paciente com adenomatose hepática, que teve indicação de transplante de fígado. 


\section{CASO CLÍNICO}

MAF, 42 anos, sexo feminino, apresentava desconforto em andar superior de abdome e história de uso prolongado de anticoncepcional. Os exames de imagem mostraram múltiplas massas hepáticas sugestivas de adenoma (figura 1). Apresentava função hepática preservada, CEA, alfa-fetoproteína e CA-19.9 dentro da normalidade e sorologias para hepatites virais negativas. Foi realizada laparotomia exploradora, com hipótese diagnóstica de adenoma hepático e com intenção de ressecção dos tumores. Durante o procedimento, foi realizado ultrassom, que evidenciou fígado esteatótico com múltiplas lesões, impossibilitando a cirurgia, devido ao comprometimento de quase todos os segmentos hepáticos. Assim sendo, foram realizadas múltiplas biópsias, que confirmaram o diagnóstico de adenoma. Foi Indicado TxF devido a desconforto abdominal (hepatomegalia), baixa qualidade de vida (impossibilitada para o trabalho), além de risco de sangramentos e degeneração maligna. A paciente foi incluída em lista de espera para TxF e permaneceu em acompanhamento ambulatorial por dois anos. Nesse período, foi avaliada a cada seis meses, não tendo apresentando aumento de alfa-fetoproteína e nem alterações radiológicas sugestivas de transformação maligna. $\mathrm{O}$ TxF ocorreu sem intercorrências. Após o exame histológico dos adenomas do fígado explantado foi diagnosticado em três deles focos de carcinoma hepatocelular, nos segmentos II, IV, VII. (figura 2). Paciente encontra-se bem 5,6 meses após o transplante, em acompanhamento ambulatorial.

Figura 1: Ressonância Nuclear Magnética, fase T2, mostrando vários nódulos (setas) hiperintensos de adenoma.

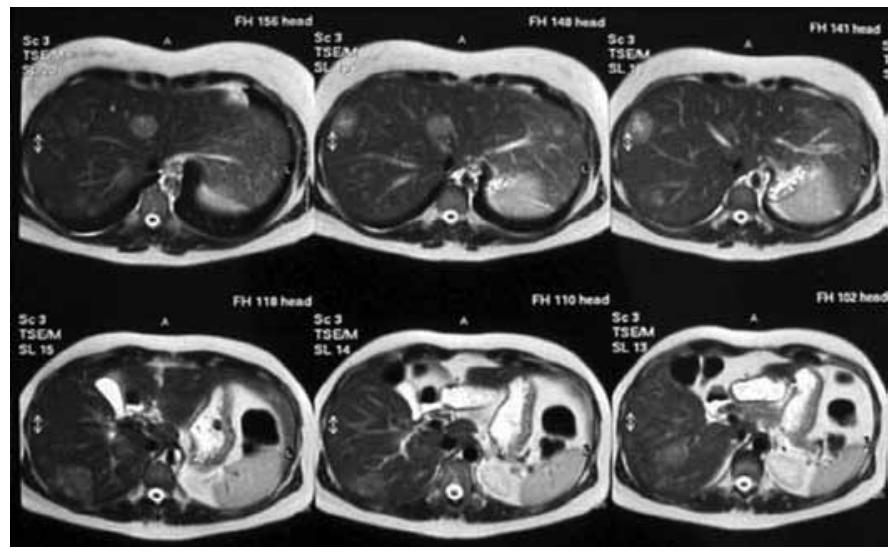

Figura 2: Exame anatomopatológico mostrando degeneração do adenoma para Hepatocarcinoma. $\mathrm{HCC}=$ hepatocarcinoma

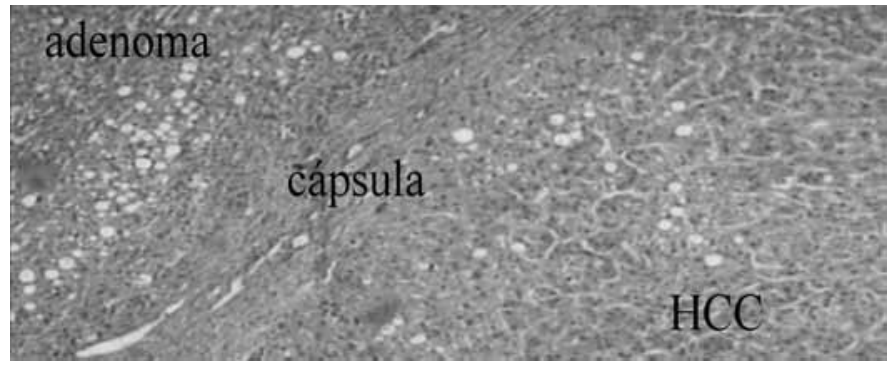

TC - Hipo/isodenso, bem delimitado, áreas heterogenias de hemorragia fibrose e necrose, RNM - Hipointenso em T1, áreas de brilho hiperintenso em T2 (hemorragia) ou moderado intensidade em T2 e arteriografia Hipervascular, enchimento centrípeto, zonas avasculares (hemorragias ou necrose), região subcapsular com vasos calibrosos (nutrícios).

\section{DISCUSSÃO}

O adenoma hepático é um tumor benigno raro de fígado e mais rara ainda é a indicação de transplante para esses pacientes. Em nossa experiência de 279 transplantes realizados no Hospital de Base da Faculdade de Medicina de São Jose do Rio Preto, essa foi a única indicação de TxF por adenomatose hepática. A sua incidência é de 3 por 1.000.000/hab por ano e mais freqiente em mulheres que fazem uso prolongado de contraceptivo oral. Nessa população, a incidência chega de um a quatro por 100.000/mulheres/ ano. $^{2,7-9}$ Esse tumor é geralmente assintomático; sua descoberta é incidental, na maioria das vezes em exame de ultrassom de rotina, mas pode apresentar-se com dor discreta em hipocôndrio direito ou dor intensa por sangramento intratumoral, complicação relatada em até $30 \%$ dos casos. ${ }^{10,11}$ Podem ainda manifesta-se como choque hipovolêmico por hemorragia em peritônio livre. Os exames de imagem podem sugerir o adenoma, porém, sendo a lesão hipervascular, o diagnóstico diferencial entre $\mathrm{HNF}$ e $\mathrm{CHC}$ é às vezes impossível. Como não se pode concluir o diagnóstico através de exames de imagem, a biópsia da lesão é necessária e, pelo risco de sangramento, não deve ser feita por via percutanêa. ${ }^{3,9,10,13,18-20}$ Concluindo o diagnóstico de adenoma, a ressecção deve ser indicada ${ }^{10,21,22}$ e, para a indicação, os seguintes critérios devem ser observados: tumor sintomático, diagnóstico incerto, paciente com desejo de engravidar e presença de complicações como hemorragia e suspeita de transformação maligna. Além disso, deve-se observar se o paciente tem baixa morbidade e se a localização da lesão é de fácil ressecção. Existem relatos de adenomas que desaparecem após cessar o uso do anticoncepcional, porém, o desenvolvimento do CHC tem sido descrito em até cinco anos após a regressão da lesão. Portanto, em casos de regressão do adenoma, se for feita opção da observação clinica, ela deve ser feita com rastreamento de $\mathrm{CHC}$ por toda a vida. Não há relato de aparecimento de $\mathrm{CHC}$ após a ressecção cirúrgica. ${ }^{9,10,23} \mathrm{O}$ risco de transformação maligna é estimado entre 5 e 18\%..$^{9,10,24}$ Embora não esteja completamente esclarecido como o adenoma evolui para $\mathrm{CHC}$, uma das explicações é que o uso prolongado do contraceptivo desenvolve foco displásico dentro do adenoma que progride para $\mathrm{CHC} .{ }^{9} \mathrm{O}$ diagnóstico e a classificação molecular do Adenoma são realizações bem recentes. Essa classificação divide os adenomas em quatro tipos, sendo que o primeiro tipo tem presença de mutação do HNF1 $\alpha$, o segundo tem mutação da $\beta$-catenina, o terceiro sem as mutações referidas, mas com inflamação e o quarto tipo também sem as mutações e sem inflamação. ${ }^{7,8}$ Essa classificação é importante, pois o adenoma que tem mutação para $\beta$-catenina tem potencial de transformação maligna. Assim sendo, num futuro próximo, provavelmente poderemos definir qual adenoma deverá ou não ser operado. Neste nosso caso, não identificamos nenhum fator que pudesse nos fazer suspeitar de CHC, como aumento da alfa feto-proteína ou alteração da imagem radiológica. A impossibilidade de hepatectomia devido aos vários nódulos maiores de $3 \mathrm{~cm}$ em fígado esteatótico, a possibilidade de sangramento e de transformação maligna associada à má qualidade de vida levaram-nos a optar pelo transplante, o qual ocorreu sem complicações, estando a paciente bem, 5,6 meses após o transplante.

Descrevemos a evolução de uma paciente portadora de adenomas hepáticos irressecáveis, que foi tratada por transplante de fígado, com sucesso. $\mathrm{O}$ exame do fígado explantado possibilitou o diagnóstico de transformação maligna para carcinomahepatocelular. 


\section{ABSTRACT}

Hepatic adenoma is a rare benign tumor of the liver which usually presented as a solitary nodule. It can be often identified in women associated to the use of oral contraceptive. Clinical presentation of such tumor can be abdominal pain at the right hypochondrium. However, it is predominantly asymptomatic. As complication, hemoperitonium, secondary to tumor rupture or malignant degeneration to hepatocellular carcinoma may occur. We describe a patient on chronic oral contraceptive use who presented multiple and unresecable adenomas associated to grade III hepatic steatosis. After a two years follow-up, she had indication for liver transplantation, due to unresecable tumor associated to abdominal discomfort and poor quality of life. The explanted liver showed incidental focus of hepatocellular carcinoma in three of the multiple adenomas in the absence of increased alpha-fetoprotein and no radiological evidence of HCC. Although the liver transplantation to treat benign tumors can be considered a radical procedure, it can be a convenient option for patients with unresecable adenomas.

Keywords: Adenoma, Liver Cell; Carcinoma, Hepatocellular; Liver Transplantation

\section{REFERÊNCIAS}

1. Herman P, Machado MA, Volpe P, Pugliese V, Vianna MR, Bacchella T, et al. Transformation of hepatic adenoma into hepatocellular carcinoma in patients with prolonged use of oral contraceptives. Rev Hosp Clin Fac Med Sao Paulo. 1994 JanFeb;49(1):30-3.

2. Ye MQ, Suriawinata A, Ben Haim M, Parsons R, Schwartz ME. A 42-year-old woman with liver masses and long-term use of oral contraceptives. Semin Liver Dis. 1999;19(3):339-44.

3. Korula J, Yellin A, Kanel G, Campofiori G, Nichols P. Hepatocellular carcinoma coexisting with hepatic adenoma - Incidental discovery after long-term oral contraceptive use. Western J Med. 1991;Oct;155:416-8

4. Weimann A, Fronhoff K, Gratz KF, Maschek H, Bartels M, Klempnauer J, et al., Diagnostic and therapeutic strategies in hepatocellular adenoma. Zentralbl Chir. 1998;123(2):140-4

5. Gossmann J, Scheuermann EH, Frilling A, Geiger H, Dietrich CF. Multiple adenomas and hepatocellular carcinoma in a renal transplant patient with glycogen storage disease type 1a (von Gierke disease). Transplantation. 2001 Jul 27;72(2):343-4.

6. Chuang WY, Chen TC, Hsu HL, Lee WC, Jeng LB, Huang SF. Liver cell adenoma with concomitant hepatocellular carcinoma: report of two cases. J Formos Med Assoc. 2002 Nov;101(11):798-802.

7. Bioulac-Sage P, Balabaud C, Bedossa P, Scoazec JY, Chiche L, Dhillon AP, at al Pathological diagnosis of liver cell adenoma and focal nodular hyperplasia: Bordeaux update. J Hepatol. 2007 Mar;46(3):521-7. Epub 2007 Jan 2.

8. Bioulac-Sage P, Blanc JF, Rebouissou S, Balabaud C, Zucman-Rossi J. Genotype phenotype classification of hepatocellular adenoma. World 2007 May 21;13(19):264954.

9. Barthelmes L, Tait IS. Liver cell adenoma and liver cell adenomatosis. HPB (Oxford). 2005;7(3):186-96.

10. Cho SW, Marsh JW, Steel J, Holloway SE, Heckman JT, Ochoa ER, at al. Surgical management of hepatocellular adenoma: take it or leave it? Ann Surg Oncol. 2008 Oct;15(10):2795-803. Epub 2008 Aug 12.

11. Choe MS, Yu ES. Hepatocellular carcinoma arising in hepatocellular adenoma. Taehan Kan Hakhoe Chi. 2002 Mar; 8(1):107-9.

12. Ferrell LD. Hepatocellular carcinoma arising in a focus of multilobular adenoma. A case report. Am J Surg Pathol. 1993 May;17(5):525-9.

13. Toso C, Majno P, Andres A, Rubbia-Brandt L, Berney T, Buhler L, et al. Management of hepatocellular adenoma: Solitary-uncomplicated, multiple and ruptured tumors. World J Gastroenterol 2005; 11(36): 5691-5.

14. Ji HF, Wang WL, Shen Y, Zhang M, Liang TB, Wu J, at al. Reduced-size liver transplantation for glycogen storage disease. Hepatobiliary Pancreat Dis Int. 2009 Feb;8(1):106-8.

15. Santambrogio R, Marconi AM, Ceretti AP, Costa M, Rossi G, Opocher E. Liver transplantation for spontaneous intrapartum rupture of a hepatic adenoma. Obstet Gynecol. 2009 Feb;113(2 Pt 2):508-10.

16. Carreiro G, Villela-Nogueira CA, Coelho HS, Basto S, Pannain VL, Caroli-Bottino A, at al. Orthotopic liver transplantation in glucose-6-phosphatase deficiency--Von Gierke disease--with multiple hepatic adenomas and concomitant focal nodular hyperplasia. J Pediatr Endocrinol Metab. 2007 Apr;20(4):545-9.

17. Schwartz ME, Roayaie S, Konstadoulakis MM, Gomatos IP, Miller CM. The Mount Sinai experience with orthotopic liver transplantation for benign tumors: brief report and literature review: case reports. Transplant Proc. 2008 Jun;40(5):1759-62.

18. Ito M, Sasaki M, Wen CY, Nakashima M, Ueki T, Ishibashi H, et al. Liver cell adenoma with malignant transformation: a case report. World J Gastroenterol. 2003 Oct;9(10):2379-81.

19. Gyorffy EJ, Bredfeldt JE, Black WC. Transformation of hepatic cell adenoma to hepatocellular carcinoma due to oral contraceptive use. Ann Intern Med. 1989 Mar $15 ; 110(6): 489-90$.

20. Huppertz A, Haraida S, Kraus A, Zech CJ, Scheidler J, Breuer J, et al. Enhancement of focal liver lesions at gadoxetic acid-enhanced MR imaging: correlation with histopathologic findings and spiral CT--initial observations. Radiology. 2005 Feb;234(2):468-78

21. Von Riedenauer WB, Shanti CM, Abouljoud MS. Resection of giant liver adenoma in a 17-year-old adolescent boy using venovenous bypass, total hepatic vascular isolation, and in situ cooling. J. Pediatr Surg. 2007 Aug;42(8):E23-7.

22. Chaib E, Gama-Rodrigues J, Ribeiro MA Jr, Herman P, Saad WA. Hepatic adenoma. Timing for surgery. Hepatogastroenterology. 2007 Jul-Aug;54(77):1382-7.

23. Bühler H, Pirovino M, Akobiantz A, Altorfer J, Weitzel M, Maranta E, et al. Regression of liver cell adenoma. A follow-up study of three consecutive patients after discontinuation of oral contraceptive use. Gastroenterology. 1982 Apr;82(4):77582 .

24. Micchelli ST, Vivekanandan P, Boitnott JK, Pawlik TM, Choti MA, Torbenson $\quad$ M. Malignant transformation of hepatic adenomas. Mod Pathol. 2008 Apr;21(4):491-7. Epub 2008 Feb 1 